PROCEEDINGS OF THE AMERICAN MATHEMATICAL SOCIETY

Volume 124, Number 10, October 1996

\title{
ON A PATTERN OF REFLEXIVE OPERATOR SPACES
}

\author{
LIFENG DING \\ (Communicated by Palle E. T. Jorgensen)
}

\begin{abstract}
A linear subspace $M$ is a separating subspace for an operator space $S$ if the only member of $S$ annihilating $M$ is 0 . It is proved in this paper that if $S$ has a strictly separating vector $x$ and a separating subspace $M$ satisfying $S x \cap[S M]=\{0\}$, then $S$ is reflexive. Applying this to finite dimensional $S$ leads to more results on reflexivity. For example, if $\operatorname{dim} S=n$, and every nonzero operator in $S$ has rank $>n^{2}$, then $S$ is reflexive.
\end{abstract}

\section{INTRODUCTION}

Let $V$ be a vector space over a field $F$, and $L(V)$ be the collection of all linear transformations on $V$. A vector $x \in V$ is a separating vector for a linear subspace $S \subset L(V)$ if its evaluation map $E_{x}: S \rightarrow S x$ defined by $s \mapsto s x$ is injective. It is proved in [6] that if $S$ is $n$-dimensional (or of denumerable Hamel basis), then each one-dimensional affine subspace in $V$ either contains no separating vector for $S$ or all but at most $n$ vectors (or countably many) in it separates $S$. Now suppose $S$ has a separating vector $x$, and $F$ has a cardinality of continuum. Then for each vector $y \in V$ there must exist some scalar $\lambda$ such that $y+\lambda x$ separates $S$. A careful review of [4] reveals the fact that for some appropriately chosen $\lambda$ the separating vector $y+\lambda x$ preserves a disjoint property, that is, if $S x$ is disjoint with a linear subspace of $V$, then $S(y+\lambda x)$ is also disjoint with the same subspace. In this note we will show that making full use of this observation leads to some results relating to reflexive operator space problems. These results are particularly useful for finite dimensional $S$. For example, we show that if every nonzero operator in $S$ has rank greater than the square of the dimension of $S$, then $S$ is reflexive. Recall that the well-known Larson's result [11] states that if $S$ is finite dimensional, then ref $S=S+\operatorname{ref} S_{F}$, where ref $S$ is the reflexive closure of $S$ and $S_{F}$ is the collection of all finite rank operators in $S$. Its immediate consequence is that $S$ is reflexive if and only if $S_{F}$ is reflexive. So the above result suggests that the obstructions to reflexivity come from small rank operators. We begin our discussion with a proof of the fact.

Proposition 1.1. Suppose $S$ is a linear subspace of $L(V)$, and the dimension of $S$ is less than the cardinality of $F$. Let $x$ be a separating vector for $S$, and let $W$ be a linear subspace of $V$ satisfying $S x \cap W=\{0\}$. Then for each vector $y \in V$ there is a scalar $\lambda \in F$ such that $y+\lambda x$ separates $S$ and $S(y+\lambda x) \cap W=\{0\}$.

Received by the editors October 14, 1994 and, in revised form, March 30, 1995.

1991 Mathematics Subject Classification. Primary 47D15; Secondary 15A30.

Key words and phrases. Reflexive operator space, separating vector, separating space.

(C)1996 American Mathematical Society 
Proof. Let $P$ be the projection onto $S x$ along a vector space complement of $S x$ that contains $W$. For an arbitrarily fixed vector $y \in V$, we define $\phi=E_{x}^{-1} \cdot P \cdot E_{y}$, where $E_{x}^{-1}$ is the inverse map of $E_{x}$. Then $\phi$ is a linear transformation from $S$ to $S$, and $P(s y)=\phi(s) x$ for each $s \in S$. Since $\operatorname{dim} S<$ cardinality of $F$, there is a scalar $-\lambda \in F$ which is not an eigenvalue of $\phi$. We show that $y+\lambda x$ separates $S$. Indeed, let $s \in S$ with $s(y+\lambda x)=0$. Then $P(s(y+\lambda x))=0$. That is, $P(s y)+\lambda s x$ $=0$. It follows that $(\phi+\lambda I)(s) x=0$. Since $x$ separates $S,(\phi+\lambda I)(s)=0$. Note that $-\lambda$ is not an eigenvalue of $\phi$, hence $s=0$.

Next, let $s \in S$ with $s(y+\lambda x) \in W$. Since $S x \cap W=\{0\}, P(s(y+\lambda x))=0$. Repeating the argument of the last paragraph leads to $s=0$. Hence $S(y+\lambda x) \cap W=$ $\{0\}$.

A linear subspace $M$ is said to be a separating subspace for $S$, if the only member $s \in S$ satisfying $s(M)=\{0\}$ is $s=0$. It is easy to see that if $S$ has a separating vector $x$, then any subspace containing $x$ is a separating subspace for $S$. But if $S$ has a separating subspace whose dimension is greater than 1, $S$ may not have a separating vector. $S$ always has a trivial separating subspace: $V$. In general, if $M$ is a separating subspace for $S$, then any subspace containing $M$ is a separating subspace for $S$.

We define $\operatorname{ref}_{a} S=\{t \in L(V): t x \in S x$ for all $x \in V\}$. If $r e f_{a} S=S, S$ is said to be algebraically reflexive [7], [11].

Theorem 1.2. Suppose linear subspace $S \subset L(V)$ has a separating vector $x$ and a separating subspace $M$ satisfying

(1) $\operatorname{dim} S<$ cardinality of $F$;

(2) $S x \cap S M=\{0\}$.

Then $S$ is algebraically reflexive.

Proof. Let $t \in r e f_{a} S$. For an arbitrarily chosen vector $y \in V$, by Proposition 1.1, there is a scalar $\lambda_{y}$ such that $y+\lambda_{y} x$ separates $S$ and $S\left(y+\lambda_{y} x\right) \cap S M=\{0\}$. Choose $a \in S$ with $t x=a x$, and choose $b \in S$ with $t\left(y+\lambda_{y} x\right)=b\left(y+\lambda_{y} x\right)$. For each vector $u \in M$, since $S u \subset S M, S x \cap S u=\{0\}$ and $S\left(y+\lambda_{y} x\right) \cap S u=\{0\}$. By Lemma 2.4 in [4], tu $=a u$ and $t u=b u$. Hence $(a-b) u=0$ holds for each $u \in M$. Since $M$ is a separating subspace for $S, a=b$. Thus $b$ is independent of the vector $y$, i.e., $t\left(y+\lambda_{y} x\right)=a\left(y+\lambda_{y} x\right)$ for each $y \in V$. It follows that $t y=a y$ for each $y \in V$. Therefore $t=a \in S$.

We recover a result in [4] as a special case:

Corollary 1.3 [4, Theorem 2.1]. Suppose linear subspace $S \subset L(V)$ has a pair of separating vectors $x$ and $y$ satisfying

(1) $\operatorname{dim} S<$ cardinality of $F$;

(2) $S x \cap S y=\{0\}$.

Then $S$ is algebraically reflexive.

\section{Finite dimensional operator SPACES}

The original notion of reflexivity was introduced in the normed space setting. We use $[\cdot]$ to denote the norm closure of a subset of a normed space $X$. For a linear subspace $S \subset B(X)$ we define

$$
\text { ref } S=\{t \in B(X): t x \in[S x] \text { for all } x \in X\} .
$$


If ref $S=S, S$ is said to be reflexive [2], [8], [11]. In particular, if $S$ is a unital algebra, then ref $S=$ alglat $S$, where alglat $S$ is the algebra of continuous linear operators that leave invariant all $S$-invariant subspaces. When $S$ is finite dimensional, ref $S=\operatorname{ref} f_{a} S$, and there is no difference between the notions reflexive and algebraically reflexive. Since we are considering the applications of the results in the last section to a finite dimensional $S$, throughout this section we still keep our discussion in the algebraic setting and assume $V$ is a vector space over a field $F$ whose cardinality is infinite. We use the terminology reflexive instead of algebraically reflexive.

Theorem 2.1. Suppose $S$ is a finite dimensional linear subspace of $L(V)$ and $\operatorname{dim} S=n$. If every nonzero operator in $S$ has rank greater than or equal to $n$, then $S$ has a separating vector.

Proof. We prove it by induction. $n=1$ is trivial. Assume the statement is true for $(n-1)$-dimensional subspace of $L(V)$. Now consider $n$-dimensional $S \subset L(V)$. Let $s_{1}, \cdots, s_{n}$ be a basis of $S$. Write $S_{(n-1)}$ as the linear span of $\left\{s_{1}, \cdots, s_{n-1}\right\}$. By the induction assumption, $S_{(n-1)}$ has a separating vector $x$. If $s_{n} x$ and $s_{1} x, \cdots, s_{n-1} x$ are linearly independent, then $x$ is a separating vector for $S$, and the proof is completed. So we assume that $s_{n} x=\alpha_{1} s_{1} x+\cdots+\alpha_{n-1} s_{n-1} x$. Replacing $s_{n}$ by $s_{n}^{\prime}=s_{n}-\alpha_{1} s_{1}-\cdots-\alpha_{n-1} s_{n-1}$, we may as well assume $s_{n} x=0$.

Since rank $s_{n} \geq n$ and $\operatorname{dim} S_{(n-1)} x=n-1$, there is a vector $y \in H$ such that $s_{n} y \notin S_{(n-1)} x$. Hence the one-dimensional linear subspace $\left\langle s_{n} y\right\rangle$, spanned by $s_{n} y$, and $S_{(n-1)} x$ are disjoint. By Proposition 1.1, there is a scalar $\lambda$ such that $y+\lambda x$ separates $S_{(n-1)}$, and $S_{(n-1)}(y+\lambda x)$ and $\left\langle s_{n} y\right\rangle$ are disjoint. We next claim that the vector $y+\lambda x$ also separates $S$.

Indeed, let $a \in S_{(n-1)}$ and $b=$ scalar multiple of $s_{n}$ with $(a+b)(y+\lambda x)=0$. Then $a(y+\lambda x)=-b(y+\lambda x)=-b y$. Since $S_{(n-1)}(y+\lambda x)$ and $\left\langle s_{n} y\right\rangle$ are disjoint, $a(y+\lambda x)=0$ and $b y=0$. Since $y+\lambda x$ separates $S_{(n-1)}, a=0$. Note that $s_{n} y \neq 0$, so $b=0$. Hence the vector $y+\lambda x$ is a separating vector for $S$.

Remark 2.2. The main result in [1] states that if linear operators $T_{1}, \cdots, T_{n}$ acting between complex vector spaces $X$ and $Y$ have a property that for every vector $\xi \in X$ the vectors $T_{1} \xi, \cdots, T_{n} \xi$ are linearly dependent, then there exists a nontrivial linear combination of these operators having rank $\leq n-1$. This result is equivalent to Theorem 2.1, where the underlying field is not necessarily complex numbers. The proof of Theorem 2.1 is different from that of [1].

Theorem 2.3. Let $S$ be a finite dimensional linear subspace of $L(V)$, and $\operatorname{dim} S=$ $n$. If every nonzero operator in $S$ has rank greater than $n^{2}$, then $S$ is reflexive.

Proof. By Theorem 2.1, $S$ has a separating vector $x$. Let $W$ be a vector space complement of $S x$ in $V$. Let $U=\{u \in V: S u \subset W\}$. Note that $\operatorname{dim} S x=n$, $\operatorname{cod}_{V} W=n$, and $\operatorname{cod}_{V} U \leq\left(\operatorname{cod}_{V} W\right) \operatorname{dim} S=n^{2}$. Let $E$ be the vector space complement of $U$ in $V$; then $\operatorname{dim} E \leq n^{2}$. We claim $U$ is a separating subspace for $S$. Indeed, let $a \in S$ with $a U=\{0\}$. The restriction of $a$ to $E$ is denoted as $\left.a\right|_{E}$. Since $U \oplus E=V$, rank $a=\left.\operatorname{rank} a\right|_{E} \leq n^{2}$. Note that if we assume rank $a>n^{2}$, we get a contradiction except for $a=0$. Hence $U$ is a separating subspace for $S$.

Since $S U \subset W$ and $W \cap S x=\{0\}, S x$ and $S U$ are disjoint. By Theorem 1.2, $S$ is reflexive.

Remark. Theorem 2.3 improves Theorem 4.3 of [4]. 
We write $S^{(n)}=\left\{s^{(n)} \in L\left(V^{(n)}\right): s \in S\right\}$ as the $n$-fold ampliation of $S$, where $s^{(n)}$ is the direct sum of $n$ copies of the operator $s$ acting on $V^{(n)}=V \oplus \cdots \oplus V$. $S$ is said to be $n$-reflexive if and only if $S^{(n)}$ is reflexive. $S$ is $n$-reflexive implies $S$ is $(n+1)$-reflexive, not vice versa [2].

Proposition 2.4 [6, Proposition 9]. The following are equivalent:

(1) $S$ has a rank-n operator $T$ in $L(V)$ for which the map $s \mapsto s T$ is injective.

(2) $S$ has a separating subspace whose dimension is at most $n$.

(3) The $n$-fold ampliation $S^{(n)}=\{s \oplus \cdots \oplus s: s \in S\}$ acting on $V^{(n)}=\{V \oplus$ $\cdots \oplus V\}$ has a separating vector.

Theorem 2.5. Suppose $S$ is finite dimensional linear subspace of $L(V)$. If $S$ has a $k$-dimensional separating subspace, then $S$ is $(k+1)$-reflexive.

Proof. Let $M$ be the $k$-dimensional separating subspace. By Proposition $2.4, S^{(k)}$ has a separating vector $x$ in $V^{(k)}$. Let $y=x \oplus 0 \in V^{(k+1)}$. Then $y$ is a separating vector for $S^{(k+1)}$. Let $E=\{0 \oplus \cdots \oplus 0 \oplus m: m \in M\} \subset V^{(k+1)}$. Then $E$ is a separating subspace for $S^{(k+1)}$. Clearly, $S^{(k+1)} y \cap S^{(k+1)} E=\{0\}$. By Theorem $1.2, S^{(k+1)}$ is reflexive, and $S$ is $(k+1)$-reflexive.

It is easy to see that the above results hold for Hilbert space, or for any locally convex space. Magajna proved in [13, Lemma 4.2] that for any $n$-dimensional subspace $S \subset B(H)$, where $H$ is a Hilbert space and $n \geq 2$, either there exists an $(n-1)$-dimensional separating subspace for $S$, or $\operatorname{dim}[S H]=1$. Note that if $\operatorname{dim}[S H]=1$, then $S$ is reflexive. So applying Theorem 2.5 we take a shortcut to a proposition that is given in [13].

Proposition 2.6 [13, Proposition 4.3]. Every n-dimensional linear subspace $S$ of $B(H)$ is at least $n$-reflexive.

We next adapt the results in Section 1 to a Hilbert space setting.

\section{Adaptation to Hilbert space operators}

Let $H$ be a Hilbert space, and let $B(H)$ be the algebra of all bounded linear operators on $H$. A vector $x \in H$ is said to be a strictly separating vector for a norm closed linear subspace $S \subset B(H)$ if the evaluation map $E_{x}$ is injective and bounded below. By the open mapping theorem, it is easy to see that $E_{x}$ has a bounded inverse $E_{x}^{-1}$. It follows that $x$ strictly separates $S$ if and only if $x$ separates $S$ and $S x$ is norm closed.

In [5] we defined the strong disjointness of two norm closed linear subspaces of a Banach space. In the Hilbert space setting we have a greater number of equivalent conditions for the definition. Although these conditions are well known, we list them and give a brief proof below for the completeness of the paper.

Proposition 3.1. Let $M$ and $N$ be norm closed nontrivial linear subspaces of $H$. Then the following conditions are equivalent:

(1) There is a positive constant $\epsilon$ such that $\|x-y\| \geq \epsilon$ for all unit vectors $x, y$ in $M$ and $N$ respectively.

(2) There is a positive number $\delta<1$ satisfying $|\langle x, y\rangle| \leq \delta$ for all unit vectors $x, y$ in $M$ and $N$ respectively.

(3) There is a positive constant $\alpha$ such that $\|x+y\| \geq \alpha(\|x\|+\|y\|)$, for all vectors $x, y$ in $M$ and $N$ respectively. 
(4) $M \cap N=\{0\}$, and let $M \oplus N$ be external sum, $M+N$ be internal sum. The map $f: M \oplus N \rightarrow M+N$ by $x \oplus y \mapsto x+y$ is bounded below.

(5) $M \cap N=\{0\}$, and $M+N$ is norm closed.

(6) There is an idempotent operator $P$ with $M=$ range $P$ and $N \subset$ kernel $P$.

Proof. (1) $\Longrightarrow$ (2) For $x \in M, y \in N$ with $\|x\|=\|y\|=1$, let $\theta$ be the argument of $\langle x, y\rangle$. Then $2(1-|\langle x, y\rangle|)=\left\|e^{i \theta} x-y\right\|^{2} \geq \epsilon^{2}$. Take $\delta=1-\epsilon^{2} / 2$.

$(2) \Longrightarrow$ (3) Pick any positive number $\alpha \leq \sqrt{(1-\delta) / 2}$.

(3) $\Longrightarrow$ (4) First part $M \cap N=\{0\}$ is trivial, and the second part is due to

$$
\|f(x \oplus y)\|=\|x+y\| \geq \alpha(\|x\|+\|y\|) \geq \alpha\|x \oplus y\| .
$$

(4) $\Longrightarrow$ (5) Let $\left\{x_{n}\right\} \subset M$ and $\left\{y_{n}\right\} \subset N$ such that $x_{n}+y_{n} \rightarrow z$. (4) implies that both $\left\{x_{n}\right\}$ and $\left\{y_{n}\right\}$ are Cauchy sequences, and it follows that $z \in M+N$.

(5) $\Longrightarrow(6)$ Since $M+N$ is closed, $H=(M+N)+(M+N)^{\perp}=M+$ $\left(N+M^{\perp} \cap N^{\perp}\right)$. Define

$$
P(x)= \begin{cases}x, & x \in M \\ 0, & x \in N+M^{\perp} \cap N^{\perp} .\end{cases}
$$

(6) $\Longrightarrow(1)$ Let unit vectors $x \in M$ and $y \in N$. Since $M \neq\{0\}, P \neq 0$, and $\|x-y\| \geq \frac{1}{\|P\|}\|P(x-y)\|=\frac{1}{\|P\|}\|P x\|=\frac{1}{\|P\|}\|x\|=\frac{1}{\|P\|}$.

Two closed linear subspaces $M$ and $N$ satisfying any one of the six conditions are called strongly disjoint. In view of the condition (2), this means $M$ and $N$ are not tangent to each other. As is discussed in [10, Problem 52], that $M$ and $N$ are strongly disjoint implies that $M$ and $N$ are disjoint, i.e., $M \cap N=\{0\}$. Not vice versa.

Theorem 3.2. Suppose $S$ is a norm closed linear subspace of $B(H)$ and $x$ is a strictly separating vector for $S$. Let $W$ be a closed linear subspace of $H$ such that $S x$ and $W$ are strongly disjoint. Then for each vector $y \in H$, there is a scalar $\lambda$ so that the vector $y+\lambda x$ is also a strictly separating vector for $S$, and $S(y+\lambda x)$ and $W$ are strongly disjoint.

Proof. If $y$ and $x$ are linearly dependent, there is nothing to prove. Assume $y$ and $x$ are linearly independent, and let $P$ be an idempotent operator with range $P=S x$ and $W \subset$ kernel $P$. We define $\phi=E_{x}^{-1} \cdot P \cdot E_{y}$ that is a bounded linear operator from $S$ to $S$. Choose $-\lambda$ in the resolvent set of the operator $\phi$. We claim that vector $y+\lambda x$ meets the requirement. Indeed, for each $s \in S,\|s(y+\lambda x)\| \geq \frac{1}{\|P\|}\|P(s(y+\lambda x))\|$. Since $P(s(y+\lambda x))=P(s y)+\lambda s x=(\phi+\lambda I)(s) x=E_{x}((\phi+\lambda I)(s)),\|P(s(y+\lambda x))\| \geq$ $\frac{1}{\left\|E_{x}^{-1}\right\|}\|(\phi+\lambda I)(s)\| \geq \frac{1}{\left\|E_{x}^{-1}\right\|\left\|(\phi+\lambda I)^{-1}\right\|}\|s\|$. Let $\alpha=\frac{1}{\|P\|\left\|E_{x}^{-1}\right\|\left\|(\phi+\lambda I)^{-1}\right\|}$. It follows that $\|s(y+\lambda x)\| \geq \alpha\|s\|$ for each $s \in S$, and the vector $y+\lambda x$ is a strictly separating vector for $S$.

Finally, for each $s \in S$ and $u \in W$, note that $P(s(y+\lambda x)-u)=P(s(y+\lambda x))$, and as follows from the last paragraph $\|s(y+\lambda x)-u\| \geq \frac{1}{\|P\|}\|P(s(y+\lambda x)-u)\|$ $=\|P(s(y+\lambda x))\| \geq \beta\|s\| \geq \frac{\beta}{\|y+\lambda x\|}\|s(y+\lambda x)\|$, where $\beta=\frac{1}{\left\|E_{x}^{-1}\right\|\left\|(\phi+\lambda I)^{-1}\right\|}$. In particular, for a unit vector $s(y+\lambda x) \in S(y+\lambda x)$ and a unit vector $u \in W$, we have $\|s(y+\lambda x)-u\| \geq \epsilon$, where $\epsilon=\frac{\beta}{\|y+\lambda x\|}>0$. Therefore, $S(y+\lambda x)$ and $W$ are strongly disjoint. 
In the following text the terminology reflexive assumes its original sense as mentioned in Section 2.

Theorem 3.3. Let $S$ be a norm closed linear subspace of $B(H)$. Suppose $S$ has a strictly separating vector $x$ and a separating subspace $M$ such that $S x$ and $[S M]$ are strongly disjoint. Then $S$ is reflexive.

Proof. Let $t \in \operatorname{ref} S$. For an arbitrarily fixed vector $y$, by Theorem 3.2, there is a scalar $\lambda$ such that $y+\lambda_{y} x$ strictly separates $S$, and $S\left(y+\lambda_{y} x\right)$ and $[S M]$ are strongly disjoint. Choose $a \in S$ with $t x=a x$, and choose $b \in S$ with $t\left(y+\lambda_{y} x\right)$ $=b\left(y+\lambda_{y} x\right)$. For each vector $u \in M$, since $S u \subset[S M],[S u]$ is strongly disjoint with $S x$ and $S\left(y+\lambda_{y} x\right)$. By Lemma 14 of [5], $t u=a u$ and $t u=b u$ respectively. Hence $(a-b) u=0$, for all $u \in M$. Since $M$ separates $S, a=b$. This means that $t\left(y+\lambda_{y} x\right)=a\left(y+\lambda_{y} x\right)$ for any $y \in H$. It follows that $t y=a y$ for all $y \in H$, and hence $t=a \in S$.

In the Hilbert space setting, we also recover a main result in [5] as a special case.

Corollary 3.4 [5, Theorem 15]. Let $S$ be a norm closed linear subspace of $B(H)$. Suppose $S$ has a strongly disjoint pair of strictly separating vectors. Then $S$ is reflexive.

Lemma 3.5. Suppose $S$ is a linear subspace of $B(H)$, and $t \in B(H)$. If $S$ has a strictly separating vector $x$, and $t y \neq 0$ for some vector $y$, then the two-dimensional linear space, $\langle x, y\rangle$, spanned by $x$ and $y$, is a separating subspace for the linear space spanned by $S$ and $t$.

Proof. If $t x \notin S x$, then $\langle t x\rangle \cap S x=0$. For $s \in S$ and scalar $\lambda$ with $(s+\lambda t) x=0$, we have $s x=-\lambda t x$. So $s x=0$, and $\lambda t x=0$. It follows that $s=0$, and $\lambda=0$. Then $x$ is a separating vector for the span of $S$ and $t$.

Now assume $t x \in S x$. Since $S x$ is closed, $t x=a x$ for some $a \in S$. Replacing $t$ by $t-a$ we may assume $t x=0$. Let $(s+\lambda t) x=0$ and $(s+\lambda t) y=0$ for some $s \in S$ and some scalar $\lambda$. Then $s x=0$, and therefore $s=0$. It follows that $\lambda=0$. So $\langle x, y\rangle$ is a separating subspace for the span of $S$ and $t$.

Theorem 3.6. Suppose $S$ is a finite dimensional linear subspace of $B(H)$ and $\operatorname{dim} S=n$. If every nonzero operator in $S$ has rank greater than or equal to $n-1$, then $S$ is 3-reflexive.

Proof. Let $s_{1}, \cdots, s_{n-1}, s_{n}$ be a basis for $S$. Let $S_{(n-1)}$ denote the linear span of $s_{1}, \cdots, s_{n-1}$. By Theorem 2.1, $S_{(n-1)}$ has a separating vector $x$. Since $S_{(n-1)}$ is finite dimensional, $S_{(n-1)} x$ is closed and hence $x$ is also a strictly separating vector for $S_{(n-1)}$. Because $s_{n} \neq 0$, there must be a vector $y$ satisfying $s_{n} y \neq 0$. Applying Lemma 3.5 concludes that the 2-dimensional subspace spanned by $x$ and $y$ is a separating subspace for $S$. The theorem follows from Theorem 2.5.

\section{BANACH SPACE SETting}

We now update Theorem 3.3 to the Banach space setting, and we can replace the strongly disjoint requirement by disjoint in the assumption of the theorem. This work is based on the operator valued analytic mapping introduced in [3].

In this section we let $X$ be a real or complex Banach space, and let $S \subset B(X)$ be a norm closed linear subspace. Suppose $x$ is a strictly separating vector for $S$. It is proved [5] that the set of all strictly separating vectors for $S$ is an open set. 
So for an arbitrarily fixed vector $y \in X$, the set $U_{x, y}=\{\lambda \in F: x+\lambda y$ strictly separates $S$ \} is an open set in the field $F$, where $F$ may be real numbers or complex numbers. Let $t \in \operatorname{ref} S$. There is $s \in S$ with $t x=s x$. Since $t-s \in \operatorname{ref} S$ too, we may replace $t$ by $t-s$ and assume $t x=0$ as well. We fix $t$. Since for each $\lambda \in U_{x, y}, x+\lambda y$ strictly separates $S$, there is a unique operator $s \in S$ satisfying $s(x+\lambda y)=\lambda t y$. Hence the map $\phi_{y}: U_{x, y} \rightarrow S$, by $\phi_{y}(\lambda)=s$ is well defined on $U_{x, y}$. It is clear that $\phi_{y}(0)=0$, and as is proved in [3] $\phi_{y}$ is locally bounded, continuous and analytic throughout $U_{x, y}$. In particular $\phi_{y}^{\prime}(0) x=t y$. It follows that the range of $t$ is contained in $S x$ [3, Proposition 3.1], where $t \in \operatorname{ref} S$ and $t x=0$ for a strictly separating vector $x$.

Theorem 4.1. Let $S$ be a norm closed linear subspace of $B(X)$. Suppose $S$ has a strictly separating vector $x$ and a separating subspace $M$ satisfying $S x \cap[S M]=\{0\}$. Then $S$ is reflexive.

Proof. Let $t \in \operatorname{ref} S$ with $t x=0$. The proof will be completed by showing $t=0$. We first claim that $t M=\{0\}$. In view of the last paragraph [3, Proposition 3.1] for each $u \in M, t u \in S x \cap[S u] \subset S x \cap[S M]=\{0\}$. Hence the claim is established.

Since $x$ strictly separates $S, E_{x}^{-1}$ exists. For each vector $y \in X, t y \in S x$ and hence $E_{y} E_{x}^{-1}(t y) \in S y$. On the other hand, since for $\lambda \in U_{x, y}, \phi_{y}(\lambda)(x+\lambda y)=\lambda t y$, $\frac{\phi_{y}(\lambda)}{\lambda} y=\frac{1}{\lambda} t y-\frac{\phi_{y}(\lambda)}{\lambda^{2}} x$. Note that the right-hand side is always in $S x$ and $\phi_{y}^{\prime}(0)$ exists, letting $\lambda \rightarrow 0$, so $\phi_{y}^{\prime}(0) y \in S x$ too. Since $E_{x}^{-1}(t y)=\phi_{y}^{\prime}(0), E_{y} E_{x}^{-1}(t y)=$ $\phi_{y}^{\prime}(0) y \in S x$, and hence $E_{y} E_{x}^{-1}(t y) \in S x \cap S y$ holds for each $y \in X$.

Next, we fix an arbitrarily chosen vector $y \in X$. Let $u \in M$ be varying. Applying the argument in the last paragraph to the vector $u+y$ leads to $E_{u+y} E_{x}^{-1}(t(u+y)) \in$ $S x$. The first claim shows that $t u=0$, therefore $E_{u+y} E_{x}^{-1}(t y) \in S x$. Note that $E_{u} E_{x}^{-1}(t y)=E_{u+y} E_{x}^{-1}(t y)-E_{y} E_{x}^{-1}(t y)$. The right-hand side belongs to $S x$, hence $E_{u} E_{x}^{-1}(t y) \in S x \cap S u \subset S x \cap[S M]=\{0\}$. This means $E_{x}^{-1}(t y) u=0$, for all $u \in M$, i.e., the operator $E_{x}^{-1}(t y)$ which is in $S$ annihilates $M$. Thus $E_{x}^{-1}(t y)=0$. It follows that $t y=E_{x} E_{x}^{-1}(t y)=E_{x}^{-1}(t y) x=0$. The arbitrariness of $y$ implies $t=0$.

Corollary 4.2 [5, Theorem 15]. Let $S$ be a norm closed linear subspace of $B(X)$. If $S$ has a strongly disjoint pair of strictly separating vectors, then $S$ is reflexive.

Corollary 4.3. Let $S$ be a norm closed linear subspace of $B(X)$. If $S$ has a strictly separating vector $x$ and a separating vector $y$ satisfying $S x \cap[S y]=\{0\}$, then $S$ is reflexive.

We will finish the paper by giving an example which shows that in Corollary 4.2 [5, Theorem 15] we cannot replace both strictly separating vectors by separating subspaces, i.e., the pattern $S N \cap S M=\{0\}$ does not imply $S$ is reflexive, and the disjointness of $S x$ and $S M$ does play a role in the reflexivity pattern.

Example 4.4. The original form of this example is Example 4.5 of [4]. We rewrite it to provide an $S \subset M_{3}=B\left(\mathbb{C}^{3}\right)$ that has a 2-dimensional separating subspace, but has no separating vector. On the other hand, $S^{(2)}$ has separating vectors and two 2-dimensional separating subspaces, but $S^{(2)}$ is not reflexive, hence $S$ is not 2-reflexive. We let

$$
S=\left\{\left[\begin{array}{ccc}
d_{1} & a & b \\
0 & d_{2} & c \\
0 & 0 & d_{3}
\end{array}\right]: d_{1}+d_{2}+d_{3}=0, a, b, c \in \mathbb{C}\right\} \subset M_{3} .
$$


Clearly, $\operatorname{dim} S=5$, and $S$ has no separating vector. Indeed, write $s_{1}, \cdots, s_{5}$ for the basis of $S$; if $S$ has a separating vector $x$, then $s_{1} x, \cdots, s_{5} x$ would be a basis of $\mathbb{C}^{3}$. It is easy to verify that the linear space spanned by $x=\left[\begin{array}{l}0 \\ 1 \\ 0\end{array}\right]$ and $y=\left[\begin{array}{l}0 \\ 0 \\ 1\end{array}\right]$ is a separating subspace for $S$. It follows that the vector $x \oplus y$ separates $S^{(2)}$, and the linear spaces $M=\operatorname{span}\{x \oplus 0, y \oplus 0\}$ and $N=\operatorname{span}\{0 \oplus x, 0 \oplus y\}$ are two 2-dimensional separating subspaces for $S^{(2)}$ satisfying $S^{(2)} M \cap S^{(2)} N=$ $\{0\}$. Moreover, $S^{(2)}$ has more than one separating vector, and due to the finite-

dimensionalness of $S^{(2)}$, these separating vectors strictly separate $S^{(2)}$. But neither pair of these cyclic subspaces of corresponding separating vectors constitutes a disjoint pair, nor any one with $S^{(2)} M$, or $S^{(2)} N$. As it is proved in [4] $S^{(2)}$ is not reflexive.

\section{ACKNOWLEDGEMENT}

The author would like to thank David R. Larson and the Department of Mathematics, Texas A \& M University, for their support which allowed the author to participate in the Workshop and Conference in Linear Analysis and Probability in summer 1994. Part of reference [3] and this paper were presented or begun during that time. The author also thanks Edward A. Azoff for his valued advice, in particular many detailed suggestions for Proposition 3.1 and Theorem 3.2. Finally, the author would like to thank referee's suggestions to reorganize this paper in a more general algebraic setting for Section 2, and the recommendation of references [9] and [12] which are very helpful.

\section{REFERENCES}

1. B. Aupetit, An improvement of Kaplansky's lemma on locally algebraic operators, Studia Math. 88 (1988), 275-278. MR 89d:47002

2. E. A. Azoff, On finite rank operators and preannihilators, Memoirs Amer. Math. Soc. 357 (1986). MR 88a:47041

3. E. A. Azoff, L. Ding and W. R. Wogen, Separating versus strictly separating vectors, to appear in Proc. Amer. Math. Soc.. CMP 95:11

4. L. Ding, Separating vectors and reflexivity, Lin. Alg. Appl. 174 (1992), 37-52. MR 94a:47075

5. L. Ding, On strictly separating vectors and reflexivity, Integ. Equat. Oper. Th. 19 (1994), 373-380. CMP 94:15

6. W. Gong, D. R. Larson and W. R. Wogen, Two results on separating vectors, preprint.

7. D. Hadwin, Algebraically reflexive linear transformations, Lin. Multilin. Alg. 14 (1983), 225233. MR 85e: 47003

8. D. Hadwin, A general view of reflexivity, Trans. Amer. Math. Soc. 344 (1994), 325-360. MR 95f: 47071

9. D. Hadwin and E.A. Nordgren, Reflexivity and direct sums, Acta Sci. Math.(Szeged) 55 (1991), 181-197. MR 92g:47064

10. P. R. Halmos, A Hilbert space problem book, 2nd ed., Springer-Verlag, New York, 1982. MR 84e: 47001

11. D. R. Larson, Reflexivity, algebraic reflexivity, and linear interpolation, Amer. J. Math. 110 (1988), 283- 299. MR 89d:47096

12. L. Livshits, Locally finite-dimensional sets of operators, Proc. Amer. Math. Soc. 119 (1993), 165-169. MR 93k:47054

13. B. Magajna, On the relative reflexivity of finitely generated modules of operators, Trans. Amer. Math. Soc. 327 (1991), 221-249. MR 91m:47064

Department of Mathematics \& Computer Science, Georgia State University, AtLANTA, Georgia 30303-3083

E-mail address: matlfd@gsusgi2.gsu.edu 